\begin{abstract}
The paper deals with positron porosimetry (PP), which is based on positron annihilation lifetime spectroscopy (PALS). The numerical analysis of positron lifetime spectra for PP is more demanding than in most of other applications of PALS. The resulting intensity distributions of ortho-positronium (o-Ps) lifetimes are interpreted in terms of the extended Tao-Eldrup (ETE) model, which provides the dependence between the $o$-Ps lifetime and pore size. Additionally, the relation between the intensity of an $o$-Ps component and the pore volume allows obtaining pore size distribution (PSD). The value of the empirical parameter $\Delta$, which is dependent on material, can be estimated from the temperature dependence of an $o$-Ps lifetime. The most unique feature of PP among other techniques that allow determination of PSDs is its ability to perform measurements in almost any conditions. This makes this method suitable for various in situ studies. In this review article, both the capabilities and the limitations of PP are discussed. The methods to overcome some of the limitations are presented.
\end{abstract}

Key words: porous materials • positron annihilation lifetime spectroscopy (PALS) • positronium • extended Tao-Eldrup model

\section{R. Zaleski}

Department of Nuclear Methods, Institute of Physics,

Maria Curie-Skłodowska University,

1 M. Curie-Skłodowskiej Sq., 20-031 Lublin, Poland, Tel.: +48 81537 6145, Fax: +48 81537 6191,

E-mail: radek@zaleski.umcs.pl

\section{Introduction}

Applications of micro- and mesoporous materials grow in numbers and scope. Initially, they were used mostly as low-k materials [1] and an environment for catalysis [2]. Their next application of great importance was drug delivery [3]. Recently, mesoporous materials were found as promising materials for gas storage [4] and removal of contaminations [5]. All these applications require accurate knowledge of the structure, especially pore size distribution (PSD), of used materials. For this purpose, several experimental methods can be used. Among them, the most widespread one is measurement of adsorption isotherms of liquid nitrogen at $77 \mathrm{~K}$. This method is limited only to open pores, which are large enough to host a nitrogen molecule. A high vacuum and sample cooling is required before starting to measure isotherms of liquid nitrogen. This can cause structural changes in materials designed to be used at room temperature. Therefore, results of this method may not be reliable for many materials. This is also the reason why it is not suitable for any in situ measurements.

An alternative experimental technique, used to determine PSDs, which does not have such limitations, is positron annihilation lifetime spectroscopy (PALS). For the purpose of porosimetry, the probe used by PALS is a long-lived triplet state of positronium. This is a bound state of an electron and a positron with parallel spins called ortho-positronium 
(o-Ps). This pseudo-atom is formed in non-conducting solids. To survive in an electron-rich environment, $o$-Ps have to be trapped in an electron-free volume (a pore, an intermolecular volume, etc.). The lifetime of the trapped $o$-Ps shortens from 142 ns (observed in a vacuum) to the value dependent on a pore size. This is a result of the pick-off process, which consists in annihilation of a positron with an electron that has anti-parallel spin, which can be found in the pore wall. Because this method is based on properties of small subatomic particles, the range of its applicability is considerably extended toward small pore sizes compared with methods based on sorption of molecules. Positronium forms randomly in the material, thus positron porosimetry (PP) is able to characterize both open and closed pores. For the same reason, presence of any substance in pores (e.g., gas or adsorbate) does not make measurement impossible, but it only changes the shape of measured positron lifetime spectra. This makes PP suitable for carrying out in situ measurements.

PP was successfully used for characterization of various porous materials based on silica [6, 7], glass [8], polymers [9], and composites [10]. There are also multiple examples of monitoring of processes that occur inside pores, e.g., phase transitions (melting, solidification, glass transition) [11-13], filling [14] or emptying [15] of pores, adsorption and desorption [16], and thermal decomposition [17]. Moreover, it is possible to observe in situ changes in porosity under pressure $[18,19]$.

\section{From PAL spectra to PSDs}

The experimental PAL spectra (Fig. 1a) represent the probability of positron annihilation at time $t$. Because positron annihilation follows the law of radioactive decay, these spectra are the sum of exponential decay components with various lifetimes (i.e., slopes of a component) and intensities (i.e., areas under a component). Typically, no purely exponential $o$-Ps components are observed in spectra acquired for porous materials. Instead, a continuous distribution of lifetimes is present. Therefore, advanced numeri-

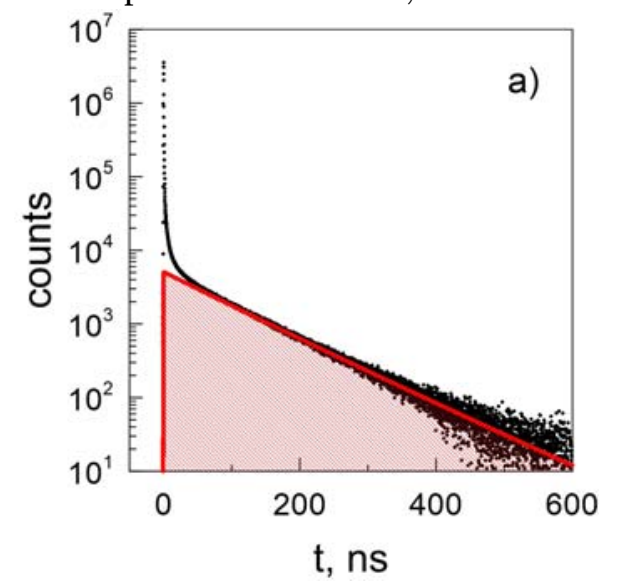

cal methods are required to reproduce the shape of a distribution of $o$-Ps lifetimes. Currently, the most assumption-free technique is MELT [21], which uses the quantified maximum entropy method to compute a distribution of an intensity over positron lifetimes (Fig. 1b). The difficulty is that MELT requires a large number of counts $\left(\sim 10^{7}\right)$ in a PAL spectrum to give reliable results [22]. Meanwhile, in some cases, the conditions of an experiment do not allow achieving a sufficient number of counts. In such a case, one can use a simplified method of analysis, which allows estimating an average lifetime, intensity, and width of a distribution that is usually sufficient to find relative changes in PSD. These parameters can be found if a lifetime distribution that has an a priori assumed shape (usually lognormal) is fitted to a spectrum using the $\chi^{2}$ minimization technique [23].

The first quantum model, used for the analysis of porous materials, was Tao-Eldrup model $[24,25]$. This model describes $o$-Ps annihilation due to the pick-off process assuming that $o$-Ps is trapped in a spherically symmetric infinite potential well and it occupies the ground level in the well. Only intrinsic $o$-Ps annihilation with a rate $\lambda_{i}=7 \mu \mathrm{s}^{-1}$ is possible in the interior of the well, where distance from its wall is larger than the empirical value $\Delta$. However, the outer part of the well represents bulk material, where $o$-Ps annihilation by pick-off occurs. Therefore, the parameter $\Delta$ allows to adjust the probability of the pick-off annihilation to fit its probability outside an actual finite potential well, which is narrower by $\Delta$ than the infinite one. The pick-off annihilation rate $\lambda_{p}$ is approximated in the model by $2 \mathrm{~ns}^{-1}$, which comes from averaging over the ortho and para states with appropriate weights. This assumption allowed finding a good correlation between the expected sizes of small free volumes and the ones calculated from model for the group of molecular crystals if the parameter $\Delta$ $=0.166 \mathrm{~nm}$ [26]. Meanwhile, thanks to examining materials with a known pore size, it turned out that surprisingly short lifetimes of $o$-Ps were observed.

A breakthrough that allowed finding agreement between PALS results and pore sizes found by adsorption methods was the development of the extended Tao-Eldrup (ETE) model [27]. A novelty in the ETE model is taking into consideration that due

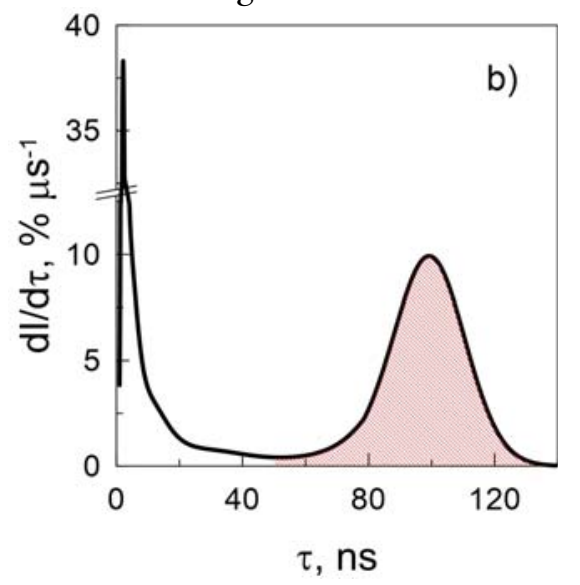

Fig. 1. Experimental positron annihilation lifetime spectrum (a) and intensity distribution of ortho-Ps lifetimes (b) of a polymer-silica composite [20]. The longest-lived component is marked by slashes. The background of random coincidences was subtracted. ortho-Ps: ortho-positronium. 
to thermal excitation, $o$-Ps in the well can occupy excited energy levels in a potential well. Because the probability of penetration of $o$-Ps into a bulk is higher at excited energy levels than at the ground energy level, this results in a greater decay constant and hence a shorter lifetime.

Pores are typically described as capillaries (infinitely long cylinders). According to the ETE model, for such geometry, the probability of pick-off process for $o$-Ps occupying the level $n m(n=1,2, \ldots, m=$ $0,1, \ldots)$ is given by

$$
P_{n m}=\frac{\int_{Z_{n m} \frac{R}{R+\Delta}}^{Z_{n m}} J_{m}(r)^{2} r d r}{\int_{0}^{Z_{n m}} J_{m}(r)^{2} r d r}
$$

where $Z_{n m}$ is the $n$-th node of the Bessel function of first kind $J_{m}(r)$ and $R$ is a pore size.

Analogously to the classic Tao-Eldrup model, the decay constant at the level $\mathrm{nm}$ is

$$
\lambda_{n m}=\lambda_{p} P_{n m}+\lambda_{i}\left(1-P_{n m}\right) .
$$

In the thermal equilibrium, the population of energy levels is given by the Boltzman distribution. Thus, the lifetime of $o$-Ps is given by

$$
\tau=\frac{\sum_{n} \sum_{m} g_{m} e^{\frac{-h^{2} Z_{n m}{ }^{2}}{16 \pi^{2} m_{e}(R+\Delta)^{2} k T}}}{\sum_{n} \sum_{m} g_{m} \lambda_{n m} e^{\frac{-h^{2} Z_{n m}^{2}}{16 \pi^{2} m_{e}(R+\Delta)^{2} k T}}}
$$

where $g_{m}$ is the statistical weight of the $m$-th state $\left(g_{0}=1, g_{m>0}=2\right), h$ is the Planck constant, $m_{e}$ is the electron mass, $k$ is the Boltzmann constant, and $T$ is a temperature.

Predictions of this model have to be calculated numerically, thus simplification thereof where infinite cylinders are replaced by infinite cuboids was proposed in Ref. [28]. Advance in computing power and numerical techniques makes such calculations fast and simple, for example, using dedicated routine EELViS [29]. The comparison of lifetimes predicted by the ETE model for room temperature with the experimental data for porous silicas and glasses shows a good agreement (Fig. 2) [28, 30, 31].

A standard result of porosimetric methods is the pore size distribution. To determine it, the knowledge of the dependence between the lifetime of $o$-Ps and the size of a free volume alone is not sufficient. It has to be complemented with the relation between the intensity of the $o$-Ps component and the pore volume. The previous analysis of this problem $[6,35]$ resulted in the development of a simple model that takes into account the probability of trapping $o$-Ps in pores of a given size and a nonlinear dependency between the pore size and the $o$-Ps lifetime in order to provide the required relation $[15,36]$.

A PSD is the pore volume per unit of pore diameter. For a cylindrical pore, it can be defined as

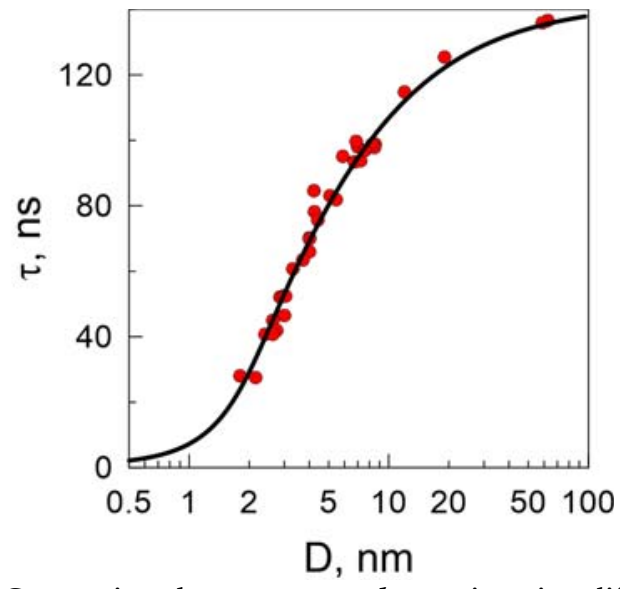

Fig. 2. Comparison between an ortho-positronium lifetime $(\tau)$ as a function of a diameter of free volume $(D)$ calculated using the extended Tao-Eldrup model for $293 \mathrm{~K}$ and the experimental data [32-34].

$$
\frac{d V}{d D} \quad \frac{d C}{d D} V(D)
$$

where $d C / d D$ is the number (concentration) of pores per unit of pore diameter and $V(D)=\pi(D / 2)^{2} L$ is the volume of a cylindrical pore with a diameter $D$ and length $L$.

The intensity of $o$-Ps that annihilates in pores with a diameter $D$ depends on the number of pores, the probability of trapping of $o$-Ps therein, and the range of positrons. An experiment provides a distribution of $o$-Ps intensity per unit of lifetime, not per unit of diameter. Therefore, the nonlinear dependence between the lifetime of $o$-Ps and the pore diameter, which is provided by the ETE model, has to be taken into account. Finally, the relation between the concentration and the intensity of $o$-Ps is given by

$$
\frac{d C}{d D} \propto \frac{\frac{d I}{d \tau} \frac{d \tau}{d D}}{\sigma(D) r}
$$

where: $d I / \mathrm{d} \tau$ is an experimental distribution of $o$-Ps intensity per unit of lifetime, $d \tau / d D$ is the derivative of the dependency obtained from the ETE model, $\sigma(D)=(2 / 3) \pi(D / 2)^{2}$ is the cross section for trapping of $o$-Ps in cylindrical pores with a diameter $D, r$ is the average range from which positrons can reach the pore.

Assuming that $r$ and $L$ do not depend on the pore diameter, the PSD can be expressed as

$$
\frac{d V}{d D} \propto \frac{d I}{d \tau} \frac{d \tau}{d D} .
$$

This very simple relation allows obtaining a PSD by multiplying the distribution of lifetimes determined in an experiment by the conversion function $d \tau / d D$, which is simply a derivative of the dependency given by the ETE model (Fig. 3a). It should be noticed that the greatest slope of the $\tau(D)$ function at ca. $2 \mathrm{~nm}$ results in the greatest precision of determination of pore size, but on the other hand, the intensity of the $o$-Ps component per unit of pore volume is the lowest in this range. For example, the same intensity of two components, which originates 

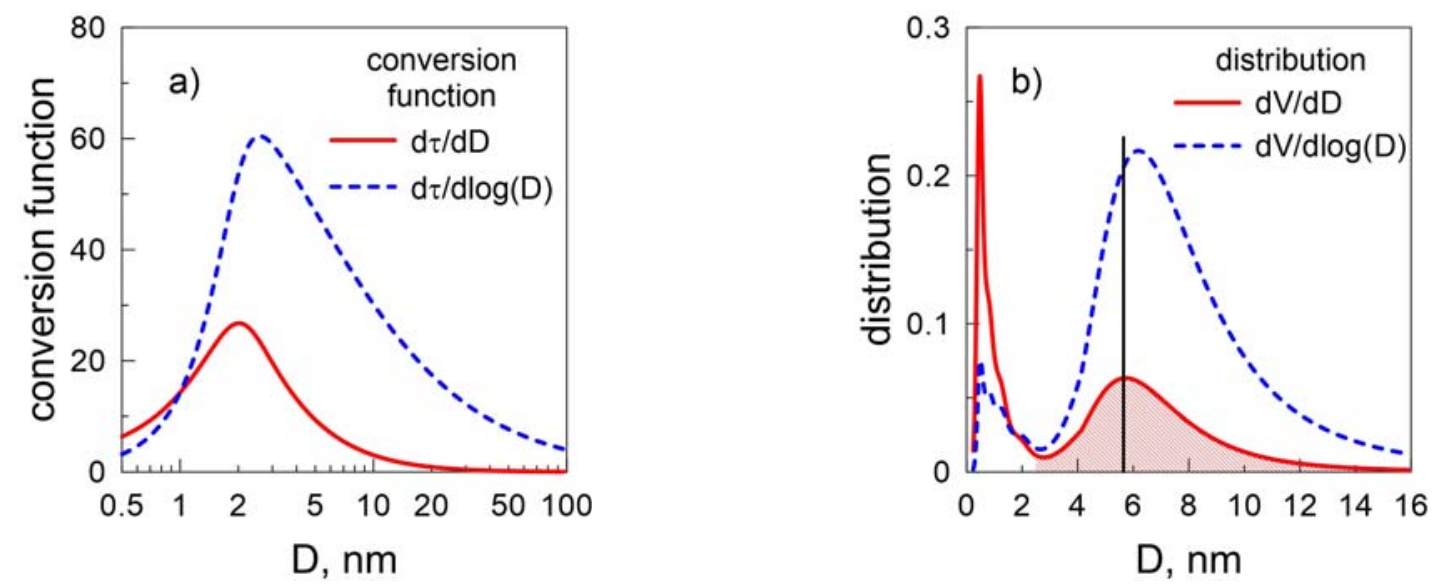

Fig. 3. Derivative of the relation between the lifetime and the pore size provided by the extended Tao-Eldrup model (a). Pore size distribution calculated for the intensity distribution of ortho-positronium lifetimes presented in Fig. 1b (b). Vertical line shows the position of the maximum of the $d V / d D$ distribution for mesopores (dashed).

from $o$-Ps annihilation in pores with sizes $2 \mathrm{~nm}$ and $5 \mathrm{~nm}$, respectively, means that the volume of the smaller pores is roughly two times bigger. For the same reason, the part of a PSD in the range of large pores, where $\tau(D)$ is almost flat, will be very low even if the intensity of the component is significant. This is the reason why $d \tau / d \log (D)$ instead of $d \tau / d D$ is frequently used as the conversion function. In particular, when a $D$ scale is logarithmic, a PSD, presented as $d V / d \log (D)$, approximately preserves the same relation between the area under the function and the pore volume in the whole range of diameters. A disadvantage of such PSD is the slightly distorted position of the maximum of a distribution (Fig. 3b).

The previously presented method for calculating PSDs requires a single empirical parameter, i.e. the broadening $\Delta$ of the potential well. It is expected that this value is not universal for all materials because it should depend on the electron density in the pore walls. Fortunately, there is a method to estimate $\Delta$ for a single sample by measurement of the temperature dependence of the $o$-Ps lifetime. If pores are large enough for $o$-Ps to populate several energy levels in a potential well, the occupation will change with

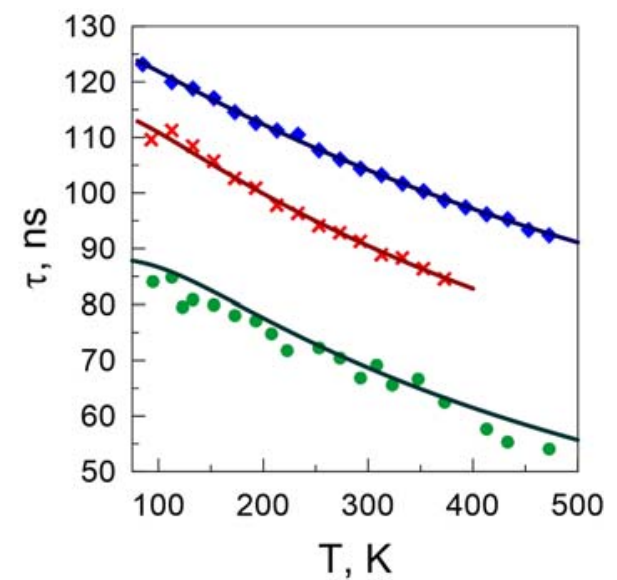

Fig. 4. Lifetime of ortho-positronium trapped in mesopores as a function of temperature in porous silica (circles) [37], silica-polymer composite (crosses) [38], and polymer (diamonds) [33]. Lines represent temperature dependences predicted by the extended Tao-Eldrup model assuming $\Delta=0.180 \mathrm{~nm}, 0.164 \mathrm{~nm}$, and $0.150 \mathrm{~nm}$, respectively. temperature and as a result, the $o$-Ps lifetime will also change. The relation between the $o$-Ps lifetime and temperature is predicted by the ETE model, thus it can be fitted to the experimental data by adjusting two parameters - the pore size $D$ and the broadening $\Delta$. The typical results are presented in Fig. 4. They show the expected dependence of $\Delta$, which is greatest in silica, smallest in polymer, and intermediate in their composite. Assumption of an incorrect value of $\Delta$ results in stretching a PSD. Thus, the distortion would be greatest for the largest pores (e.g., even $60 \%$ change in pore size per $20 \%$ change of $\Delta$ for pores greater than $10 \mathrm{~nm}$ ). Therefore, the correct $\Delta$ is critical for obtaining accurate PSDs.

\section{Applicability of PP}

PP is suitable for characterization of numerous samples, where a contribution of $o$-Ps lifetime shortening (quenching), due to effects other than pick-off, is negligible. The most common $o$-Ps quenching occurs in pores due to the ortho-para spin conversion of positronium, which is caused by paramagnetic oxygen present in the air. Therefore, it is preferable to carry out porosimetric measurements in a vacuum. Additionally, a vacuum assures that no adsorbate molecules are present on pore walls, which can distort the results [39]. Nevertheless, it is possible to modify the ETE model to take the spin conversion into account [40]. Measurements in the air have some advantages, that is, a slightly less steep $\tau(D)$ function and shorter $o$-Ps lifetimes. The first advantage makes the sensitivity of PP more uniform in the whole range of sizes. The second one makes measurement less susceptible to the nonlinearity of a PAL spectrometer and prevents disappearance of the long-lived components in the background of random coincidences. Measurements in the air also suppress another source of a possible distortion of results, namely, $o$-Ps migration from small to large pores if they are connected [41]. The migration seems to be the most common cause of disagreement between PP and other techniques. However, if better known, it would give additional information about 
pore interconnectivity, the length of pores, and the size of connections between them. Still, as there has been no systematic study of the migration yet, these properties of a sample can be deduced only qualitatively.

There are porous materials where significant deviations from expected results are observed. Particularly, at low temperature, the $\tau(T)$ relation does not follow the dependence expected by the ETE model for numerous samples [42-45]. Attempts to explain this behavior as thermal expansion, adsorption of gas remnants on pore walls, or $o$-Ps localization [46] were taken. However, its origin is unknown in many cases. Deviation of $\tau(T)$ from a model curve is highly unfavorable, because it makes the estimation of $\Delta$ and hence the determination of pore size unreliable.

The greatest drawback of PP is lack of positronium formation in conducting materials. Therefore, it cannot be used for characterization of the large group of porous materials based on metals, semiconductors, or carbon. However, it seems possible to perform such studies if the surface of these materials is covered by a nonconductive layer of an adsorbate.

\section{References}

1. Hatton, B. D., Landskron, K., Hunks, W. J., Bennett, M. R., Shukaris, D., Perovic, D. D., \& Ozin, G. A. (2006). Materials chemistry for low-k materials. Mater. Today, 9(3), 22-31. DOI: 10.1016/S13697021(06)71387-6.

2. Taguchi, A., \& Schüth, F. (2005). Ordered mesoporous materials in catalysis. Microporous Mesoporous Mater., 77(1), 1-45. DOI: 10.1016/j.micromeso.2004.06.030.

3. Slowing, I. I., Vivero-Escoto, J. L., Wu, C. -W., \& Lin, V. S. Y. (2008). Mesoporous silica nanoparticles as controlled release drug delivery and gene transfection carriers. Adv. Drug Delivery Rev., 60(11), 1278-1288. DOI: 10.1016/j.addr.2008.03.012.

4. Chang, F., Zhou, J., Chen, P., Chen, Y., Jia, H., Saad, S. M. I., Gao, Y., Cao, X., \& Zheng, T. (2013). Microporous and mesoporous materials for gas storage and separation: a review. Asia-Pac. J. Chem. Eng., 8(4), 618-626. DOI: 10.1002/apj.1717.

5. Maretto, M., Blanchi, F., Vignola, R., Canepari, S., Baric, M., Iazzoni, R., Tagliabue, M., \& Papini, M. P. (2014). Microporous and mesoporous materials for the treatment of wastewater produced by petrochemical activities. J. Clean. Prod., 77, 22-34. DOI: 10.1016/j.jclepro.2013.12.070.

6. Gidley, D. W., Frieze, W. E., Dull, T. L., Sun, J., Yee, A. F., Nguyen, C. V., \& Yoon, D. Y. (2000). Determination of pore-size distribution in low-dielectric thin films. Appl. Phys. Lett., 76(10), 1282-1284. DOI: 10.1063/1.126009.

7. Goworek, J., Zaleski, R., Borówka, A., Kusak, R., \& Kierys, A. (2009). Pore structure and morphology of mesoporous silicate and aluminosilicate molecular sieves by nitrogen adsorption, AFM and PALS. In S. Kaskel, P. Llewellyn, F. Rodriguez-Reinoso, \& N. A. Seaton (Eds.), Characterisation of porous solids VIII: Proceedings of the 8th International Symposium on the Characterisation of Porous Solids (pp. 303-310). The Royal Society of Chemistry.

8. Kullmann, J., Enke, D., Thraenert, S., Krause-Rehberg, R., \& Inayat, A. (2010). Characterization of nanoporous monoliths using nitrogen adsorption and positronium annihilation lifetime spectroscopy. Colloids Surf. A, 357(1/3), 17-20. DOI: 10.1016/j. colsurfa.2009.09.030.

9. Zaleski, R., Stefaniak, W., Maciejewska, M., \& Goworek, J. (2009). Porosity of polymer materials by various techniques. J. Porous Mat., 16(6), 691-698. DOI: $10.1007 / \mathrm{s} 10934-008-9250-7$.

10. Zaleski, R., Kierys, A., Grochowicz, M., Dziadosz, M., \& Goworek, J. (2011). Synthesis and characterization of nanostructural polymer-silica composite: Positron annihilation lifetime spectroscopy study. $J$. Colloid Interf. Sci., 358(1), 268-276. DOI: 10.1016/j. jcis.2011.03.008.

11. Kilburn, D., Sokol, P. E., Sakai, V. G., \& Alam, M. A. (2008). Confinement induces both higher free volume and lower molecular mobility in glycerol. Appl. Phys. Lett., 92(3), 033109. DOI: 10.1063/1.2835903.

12. Iskrová, M., Majerník, V., Illeková, E., Šauša, O., Berek, D., \& Krištiak, J. (2009). Free volume seen by positronium in bulk and confined molecular liquid. Mater. Sci. Forum, 607, 235-237. DOI: 10.4028/www. scientific.net/MSF.607.235.

13. Zaleski, R., \& Goworek, J. (2009) n-Nonadecane embedded in mesopores. Mater. Sci. Forum, 607, 180-182 DOI: 10.4028/www.scientific.net/MSF.607.180.

14. Kullmann, J., Enke, D., Thraenert, S., Krause-Rehberg, R., \& Beiner, M. (2012). Characterization of pore filling of mesoporous host systems by means of positronium annihilation lifetime spectroscopy (PALS). Opt. Appl., 42(2), 281-286. DOI: 10.5277/ oa120205.

15. Zaleski, R., Stefaniak, W., Maciejewska, M., \& Goworek, J. (2010). Porosity evolution of VP-DVB/ MCM-41 nanocomposite. J. Colloid Interf. Sci., 343(1), 134-140. DOI: 10.1016/j.jcis.2009.11.019.

16. Zaleski, R., Dolecki, W., Kierys, A., \& Goworek, J. (2012). $n$-Heptane adsorption and desorption on porous silica observed by positron annihilation lifetime spectroscopy. Microporous Mesoporous Mater., 154, 142-147. DOI: 10.1016/j.micromeso.2011.08.032.

17. Zaleski, R., \& Wawryszczuk, J. (2008). Positron porosimetry studies of template removal from as-synthesized MCM-41 silica. Acta Phys. Pol. A, 113(5), 1543-1550.

18. Zaleski, R., Goworek, J., \& Borówka, A. (2007). Positronium annihilation study of as-synthesized MCM-41 silica under pressure. In P. L. Llewellyn, F. Rodriquez-Reinoso, J. Rouqerol, \& N. Seaton (Eds.), Characterization of porous solids VII. (Studies in Surface Science and Catalysis, Vol. 160, pp. 471-478). Elsevier.

19. Zaleski, R., Maciejewska, M., \& Puzio, M. (2015). Mechanical stability of porous copolymers by positron annihilation lifetime spectroscopy. J. Phys. Chem. C, 119(21), 11636-11645. DOI: 10.1021/acs. jpcc.5b01722.

20. Zaleski, R., Kierys, A., Dziadosz, M., Goworek, J., \& Halasz, I. (2012). Positron annihilation and N2 -adsorption for nanopore determination in silicapolymer composites. RSC Adv., 2(9), 3729-3734. DOI: 10.1039/C2RA20147J.

21. Shukla, A., Peter, M., \& Hoffmann, L. (1993). Analysis of positron lifetime spectra using quantified maximum entropy and a general linear filter Nucl. Instrum. Methods Phys. Res. Sect. A-Accel. Spectrom. Dect. Assoc. Equip., 335(1/2), 310-317. DOI: 10.1016/0168-9002(93)90286-Q.

22. Zaleski, R. (2006). Measurement and analysis of the positron annihilation lifetime spectra for mesoporous silica. Acta Phys. Pol. A, 110(5), 729-738. 
23. Kansy, J. (1996). Microcomputer program for analysis of positron annihilation lifetime spectra. Nucl. Instrum. Methods Phys. Res., Sect. A-Accel. Spectrom. Dect. Assoc. Equip., 374(2), 235-244. DOI: 10.1016/0168-9002(96)00075-7.

24. Tao, S. J. (1972). Positronium annihilation in molecular substances. J. Chem. Phys., 56(11), 5499-5510. DOI: 10.1063/1.1677067.

25. Eldrup, M., Lightbody, D., \& Sherwood, J. N. (1981). The temperature dependence of positron lifetimes in solid pivalic acid. Chem. Phys., 63(1/2), 51-58. DOI: 10.1016/0301-0104(81)80307-2.

26. Nakanishi, H., Wang, S. J., \& Jean, Y. C. (1988). Microscopic surface tension studies by positron annihilation. In S. C. Sharma (Ed.), Proceedings of the International Symposium on Positron Annihilation Studies of Fluids (pp. 292-298). Singapore: World Scientific.

27. Ciesielski, K., Dawidowicz, A. L., Goworek, T., Jasińska, B., \& Wawryszczuk, J. (1998). Positronium lifetimes in porous Vycor glass. Chem. Phys. Lett., 289(1/2), 41-45. DOI: 10.1016/S0009-2614(98)00416-3.

28. Dull, T. L., Frieze, W. E., Gidley, D. W., Sun, J. N., \& Yee, A. F. (2001). Determination of pore size in mesoporous thin films from the annihilation lifetime of positronium. J. Phys. Chem. B, 105(20), 4657-4662. DOI: $10.1021 /$ jp004182v.

29. Zaleski, R., EELViS. http://sourceforge.net/projects/ eelvis/ Accessed: February 20, 2015.

30. Thränert, S., Enke, D., Dlubek, G., \& Krause-Rehberg, R. (2009). Positron lifetime spectroscopy on controlled pore glass porosimetry and pore size distribution. Mater. Sci. Forum, 607, 169-172. DOI: 10.4028/0-87849-348-4.169.

31. Zaleski, R., Wawryszczuk, J., \& Goworek, T. (2007). Pick-off models in the studies of mesoporous silica MCM-41. Comparison of various methods of the PAL spectra analysis. Radiat. Phys. Chem., 76(2), 243-247. DOI: 10.1016/j.radphyschem.2006.03.044.

32. Goworek, T., Ciesielski, K., Jasinska, B., \& Wawryszczuk, J. (1997). Positronium in large voids. Silicagel. Chem. Phys. Lett., 272(1/2), 91-95. DOI: 10.1016/ S0009-2614(97)00504-6.

33. Jasińska, B., \& Dawidowicz, A. L. (2003). Pore size determination in Vycor glass. Radiat. Phys. Chem., 68(3/4), 531-534. DOI: 10.1016/S0969806X(03)00224-X.

34. Śniegocka, M., Jasińska, B., Wawryszczuk, J., Zaleski, R., Deryło-Marczewska, A., \& Skrzypek, I. (2005). Testing the extended Tao-Eldrup model. Silica gels produced with polymer template. Acta Phys. Pol. A, 107, 868-873.

35. Dlubek, G., Sen Gupta, A., Pionteck, J., Hassler, R., Krause-Rehberg, R., Kaspar, H., \& Lochhaas, K.
H. (2005). Glass transition and free volume in the mobile (MAF) and rigid (RAF) amorphous fractions of semicrystalline PTFE: a positron lifetime and PVT study. Polymer, 46(16), 607-6089. DOI: 10.1016/j. polymer.2005.04.090.

36. Zaleski, R., Goworek, J., \& Maciejewska, M. (2009). Positronium lifetime in porous VP-DVB copolymer Phys. Status Solidi C, 6(11), 2445-2447. DOI: $10.1002 /$ pssc. 200982075.

37. Goworek, T., Jasinska, B., Wawryszczuk, J., Zaleski, R., \& Suzuki, T. (2002). On possible deviations of experimental PALS data from positronium pick-off model estimates. Chem. Phys., 280(3), 295-307. DOI: 10.1016/S0301-0104(02)00491-3.

38. Gorgol, M., Tydda, M., Kierys, A., \& Zaleski, R. (2012). Composition of pore surface investigated by positron annihilation lifetime spectroscopy. $\mathrm{Mi}$ croporous Mesoporous Mater., 163, 276-281. DOI: 10.1016/j.micromeso.2012.07.029.

39. Gorgol, M., Zaleski, R., \& Kierys, A. (2013). Gas filling of SBA-15 silica micropores probed by positron annihilation lifetime spectroscopy (PALS). Nukleonika, 58(1), 227-231.

40. Zaleski, R., \& Sokól, M. (2011). Influence of atmospheric gases present in the pores of MCM-41 on lifetime of ortho-positronium. Mater. Sci. Forum, 666, 123-128. DOI: 10.4028/www.scientific.net/ MSF.666.123.

41. Zaleski, R., Błażewicz, A., \& Kierys, A. (2013). Ortho-positronium migration in mesopores of MCM-41, MSF and SBA-3. Nukleonika, 58(1), 233-238.

42. Thraenert, S., Hassan, E. M., Enke, D., Fuerst, D., Krause-Rehberg, R. (2007). Verifying the RTE model: ortho-positronium lifetime measurement on controlled pore glasses. Phys. Status Solidi C, 4(10), 3819-3822. DOI: 10.1002/pssc.200675738.

43. Jasińska, B., Zaleski, R., Śniegocka, M., Reisfeld, R., \& Zigansky, E. (2007). Testing ETE model, temperature dependences of PALS data. Phys. Status Solidi C, 4(10), 3985-3988. DOI: 10.1002/pssc.200675809.

44. Śniegocka, M., Jasińska, B., Goworek, T., \& Zaleski, R. (2006). Temperature dependence of o-Ps lifetime in some porous media. Deviations from ETE model. Chem. Phys. Lett., 430(4/6), 351-354. DOI: 10.1016/j.cplett.2006.09.001.

45. Fischer, C. G., Weber, M. H., Wang, C. L., McNeil, S. P., \& Lynn, K. G. (2005). Positronium in low temperature mesoporous films. Phys. Rev. B, 71(18), 180102. DOI: 10.1103/PhysRevB.71.180102.

46. Zaleski, R. (2013). Ortho-positronium localization in pores of Vycor glass at low temperature. J. Phys. Conf. Ser., 443(1), 012062. DOI: 10.1088/1742$6596 / 443 / 1 / 012062$. 